In eigener Sache

wissen kompakt 2019 $13: 102$

https://doi.org/10.1007/s11838-019-00091-z

(c) Freier Verband Deutscher Zahnärzte (FVDZ) and Springer Medizin Verlag GmbH, ein Teil von Springer Nature 2019

\title{
Neuer Zugang zu Ihren Fortbildungen
}

\section{Wichtige Information für alle FVDZ- Mitglieder}

Um auf die Inhalte zugreifen zu können, müssen Sie sich einmalig auf SpringerZahnmedizin.de registrieren.

Wichtig Halten Sie Ihre FVDZ-Mitgliedsnummer bereit! Diese finden Sie auf Ihrem FVDZ-Mitgliedsausweis.

\section{Ich bin noch nicht auf SpringerZahnmedizin.de registriert}

FVDZ-Mitglieder, die noch nicht auf SpringerZahnmedizin.de angemeldet sind, registrieren sich bitte einmalig unter https://www.springermedizin.de/ register. Bitte geben Sie auf jeden Fall unter „Mitgliedschaften“ den Freien Verband Deutscher Zahnärzte als Ihren Verband an, und tragen Sie Ihre sechsstellige FVDZ-Mitgliedsnummer ein. Diese wird beim FVDZ anonym verifiziert. Bitte beachten Sie, dass für den Abschluss der Registrierung ein Berufsnachweis (z.B. Approbationsurkunde, Zahnarztausweis) notwendig ist. Anschließend erhalten Sie per E-Mail einen Link, mit dem Sie Ihr Profil auf SpringerZahnmedizin.de aktivieren. Über CME.SpringerZahnmedizin.de kommen Sie direkt zu den CME-Kursen aus Ihren zahnmedizinischen Zeitschriften.

\section{Ich bin bereits auf Springer- Zahnmedizin.de registriert}

Für alle FVDZ-Mitglieder, die bereits ein Nutzerkonto auf SpringerZahnmedizin.de besitzen, ändert sich auch nach der Umstellung nichts. Sie haben weiterhin freien Zugriff auf alle Ihre Inhalte.

Im seltenen Fall, dass Sie trotz $\mathrm{Re}$ gistrierung nicht auf Ihre Verbandszeitschriften zugreifen können, tragen Sie bitte Ihre FVDZ-Mitgliedsnummer in Ihrem Profil nach: Gehen Sie unter „Mein Profil“ auf „Meine Mitgliedschaften“. Wählen Sie „Freier Verband Deutscher Zahnärzte“ aus, und tragen Sie Ihre sechsstellige FVDZ-Mitgliedsnummer ein. Nach dem Speichern ist Ihr Zugriff sofort wieder frei.

Hinweis Wenn Sie nicht sofort nach der Registrierung eine E-Mail bekommen, ist sie vielleicht im Spam-Filter hängen geblieben oder die E-Mail-Adresse stimmt nicht. Bitte wenden Sie sich an kundenservice@springermedizin.de.

Ihr Freier Verband Deutscher Zahnärzte

\footnotetext{
Infobox Sie haben Fragen ...

- zu Ihrer FVDZ-Mitgliedschaft? Dann wenden Sie sich bitte an den FVDZ, Gaby Schneider, E-Mail: gs@fvdz.de, Tel.: 0228-8557-41.

- zur Teilnahme an den CME-Kursen? Dann wenden Sie sich bitte an den Springer-Kundenservice unter kundenservice@springermedizin.de oder Tel.: 0800-7780777 (kostenfrei, Mo-Fr 9-17 Uhr).
}

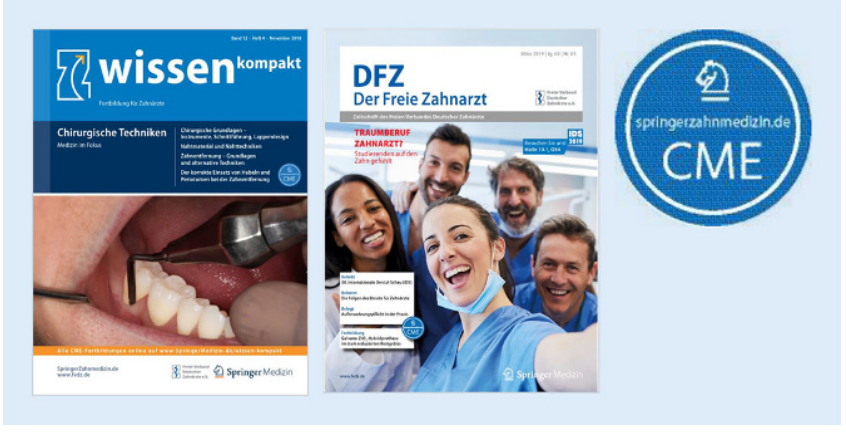

Abb. $1<$ Ab September: Neuer Zugang zur OnlineFortbildung von wissen kompakt und Der Freie Zahnarzt 